\title{
Nuevas dataciones para el Paleolítico superior de Cueva Morin (Villanueva de Villaescusa, Cantabria)
}

\author{
José Manuel Maíllo Fernández ${ }^{1}$ \\ HeLĖNe VALLADAs ${ }^{2}$ \\ Victoria Cabrera Valdés 1 \\ y Federico Bernaldo de Quirós ${ }^{3}$
}

\begin{abstract}
RESUMEN
ABSTRACT

Se presentan dos nuevas dataciones

We present the new $\mathrm{C} 14$ dates come C14 realizadas en Cueva Morin de los niveles 11 (Musteriense) y 8 from Cueva Morin samples of the 11 (Mousterian) and 8 (Archaic (Auriñaciense arcaico). También se efectúa la contextualización de éstas en el marco de la Transición entre el Paleolítico medio y el superior en la región cantábrica. Aurignacian) levels. Moreover we offer the framework of this dates for the Middle-Upper Palaeolithic Transition in the Cantabrian region.

PALABRAS CLAVE: Dataciones, C14, Cueva Morin, KEY WORDS: Musteriense Final, Auriñaciense Dates, C14, Cueva Morin, Late Mousterian, Archaic Aurignacian. arcaico.
\end{abstract}

Dpto. de Prehistoria e Historia Antigua, UNED.E-mail: jimaillo@geo.uned.es Laboratoire des Sciences du Climat et l'Environnement, CNRS.

3 Área de Prehistoria, Universidad de León. 


\section{INTRODUCCIÓN}

Cueva Morin es uno de los yacimientos clave para la comprensión del Paleolítico en el norte de la Península Ibérica. Más concretamente, su papel es vital para la comprensión de la Transición entre el Paleolítico Medio y Superior en la región cantábrica, ya que presenta una serie de niveles correspondientes al Musteriense Final, posee el nivel más característico de Chatelperroniense de nuestro país y dispone una más que aceptable secuencia de Auriñaciense (dos niveles Auriñacienses arcaicos y dos Auriñacienses antiguos).

El yacimiento se encuadra en Villanueva de Villaescusa, en una colina caliza de origen urgoniano dentro de la cuenca de drenaje del río Solía, concretamente del arroyo Obregón. Su boca se orienta al suroeste, está a 57 m.s.n.m., a $22 \mathrm{~m}$. del fondo del valle y $6 \mathrm{Km}$ al sur de la actual bahía de Santander.

La cueva, descubierta en 1910 por $\mathrm{H}$. Obermaier y $\mathrm{P}$. Wermet ha sido objeto de numerosas intervenciones entre las que debemos destacar las de J. Carballo entre 1917 y 1919 (Carballo, 1923) y las del conde de la Vega de Sella entre 1918 y 1920 de forma simultánea a las anteriores (Vega del Sella, 1921).

Las últimas excavaciones realizadas en el yacimiento fueron llevadas a cabo por un equipo hispano-estadounidense dirigido por el Prof. J. González Echegaray y L. G. Freeman, poniendo al descubierto una importante y completa estratigrafía compuesta por un nivel aziliense, uno magdaleniense, uno solutrense superior, dos gravetienses, uno auriñaciense evolucionado, dos auriñaciense antiguo, dos auriñaciense arcaico, uno chatelperroniense, siete musterienses, cuatro estériles y uno final musteriense.

Pese a ser el primer referente de la técnica arqueológica en nuestro país en cuanto a técnicas y métodos empleados, las dataciones «absolutas" no resultaron todo lo satisfactorias que cabría haber esperado, en parte por la escasa vida operativa de las técnicas de datación empleadas, el carbono-14, aún en proceso experimental (cuadro 1).

Estas dataciones han sido ya suficientemente discutidas en numerosos trabajos (p. ej. Bernaldo de Quirós, 1982; Arrizabalaga, 1995), por lo que no vamos a entrar en más detalles.

\section{LAS NUEVAS DATACIONES}

Debido a los problemas que planteaban las dataciones nos decidimos a realizar una serie de dataciones en los niveles más significativos en el de- 
Tabla 1. Dataciones de C14 de Cueva Morin (según Stuckenrath, 1978)

\begin{tabular}{cllll}
\hline Nivel & N. ${ }^{\circ}$ Lab. & \multicolumn{1}{c}{ Tipo } & Datación $($ B.C.) & Datación B.P.) \\
\hline 10 & SI-951 & Carbón & $26.660 \pm 577$ & $28.610 \pm 560$ \\
10 & SI-951A & Carbón (parte disoluble en Na OH) & $35.000 \pm 6777$ & $36.950 \pm 6580$ \\
8 a & SI-952 & Carbón & $26.485 \pm 556$ & $28.435 \pm 540$ \\
8 a & SI-952A & Carbón (parte disoluble en Na OH) & $26.205 \pm 757$ & $28.155 \pm 735$ \\
8 a & SI-956 & Carbón & $26.565 \pm 1324$ & $28.600 \pm 1285$ \\
7 & SI-955 & Carbón & $27.565 \pm 865$ & $29.515 \pm 840$ \\
7 & SI-955A & Carbón (parte disoluble en Na OH) & $26.105 \pm 1535$ & $29.055 \pm 1490$ \\
$7 / 6$ & SI-954 & Carbón & $30.465 \pm 901$ & $32.415 \pm 875$ \\
5 sup & SI-953 & Carbón & $18.760 \pm 350$ & $20.710 \pm 340$ \\
\hline
\end{tabular}

bate científico actual en el que se encuadra Cueva Morin, estos son los concernientes a la transición entre el paleolítico Medio y Superior. Por ello, tomamos dos muestras correspondientes a los niveles 11 (Musteriense) y 8 (Auriñaciense arcaico). De esta forma también ubicamos, mediante dataciones relativas, los niveles 10 (Chatelperroniense) y 9 (Auriñaciense arcaico).

La muestras de carbón fueron extraídas directamente del corte ubicado en el lado oeste de la cueva, tras la realización de una limpieza de corte y tratados por una de nosotros (H.V.) en el Laboratoire des Sciences du Climat et de l'Environnement, los resultados obtenidos se pueden comprobar en la cuadro 2.

Tabla 2. Nuevas dataciones obtenidas para Cueva Morin

\begin{tabular}{cccc}
\hline Nivel & $N^{\circ}{ }^{\circ}$ Lab. & Tipo & Datación \\
\hline 8 & GIFA -96263 & AMS & $36.590 \pm 770$ \\
11 & GIFA 96264 & AMS & $39.770 \pm 730$ \\
\hline
\end{tabular}

\section{CONTEXTUALIZACIÓN}

Las nuevas dataciones obtenidas en Cueva Morin resultan muy interesantes a la hora de contextualizar la Transición entre el paleolítico medio y el superior. En primer lugar, el Musteriense de Cueva Morin se presenta en un momento final del Musteriense; además las características tecnológicas de este nivel 11, junto con el infrapuesto 12, en los que podemos encontrar esquemas operativos discoides junto a otros de carácter laminar 
(Maíllo, 2001, 2003) plantea una interesante disyuntiva: por un lado, lo ponen en relación con los niveles, algo más antiguos de la Unidad 20 de la cueva de El Castillo desde un punto de vista tecnológico (Cabrera et alli, 2000 ). Por otro, cronológicamente estaría en relación con el nivel $18 \mathrm{c}$ de El Castillo, clasificado como Auriñaciense de Transición.

En cuanto a la datación del nivel 8 , debemos comentar que encontramos referentes cronológicos similares en el yacimiento asturiano de La Viña con una datación del nivel XIII de 36.500 B.P. Este nivel presenta una azagaya de base hendida, y numerosas hojitas Dufour y hojitas tipo Krems. Más clara parece su relación con el nivel 16 de El Castillo, con una datación de 34.000 B.P. y con unos esquemas operativos líticos muy similares. Labeko Koba presenta unas dataciones algo más tardías, pero tecnológicamente encaja perfectamente con la industria lítica de Cueva Morin.

Las dataciones para el Musteriense final y para el Auriñaciense arcaico de Cueva Morin ofrecen una datación ante $y$ post quem para el Chatelperroniense del yacimiento. Este hecho ubica a este conjunto industrial en un horquilla que varía entre 40-36550 B.P., lo que representa una datación relativamente antigua. Este hecho también ha sido constatado desde un punto de vista tecnológico por el gran peso cuantitativo de esquemas operativos de tipo discoide (Maíllo Fernández, 2003).

Por último, este panorama que hemos esbozado en los párrafos anteriores presenta una transición entre el paleolítico medio y el superior compleja, demasiado distante de la evolución lineal imperante en nuestra disciplina. Para comenzar debemos unir al Musteriense Final y al Auriñaciense arcaico, dos conjuntos industriales que podríamos denominar «transicionales" como son el Chatelperroniense y el Auriñaciense de Transición. Pero además, las pautas cronológicas de cada uno de ellos se solapan de forma muy extensa incluso dentro de la escala temporal en la que nos movemos para estos momentos históricos. Como podemos comprobar en el gráfico 1, existe un solapamiento entre los niveles Musterienses, Auriñacienses de Transición, Chatelperroniense y, con algo más de cautela, con el Auriñaciense arcaico.

Así, por ejemplo, el Musteriense de El Esquilleu (nivel XIII) es coetáneo del $18 b$ (Auriñaciense de Transición de El Castillo), mientras que los niveles XIF y VI lo son de Morin VIII o La Viña XIII. Ante esta situación podríamos argüir que se trata de una pervivencia marginal debido a la situación marginal y montañosa de El Escquilleu. Sin embargo, el Musteriense de Morin 11 es más o menos coetáneo de Castillo 18c y estos dos yacimientos se encuentran geográficamente muy próximos. Por último el Chatelperroniense de Morin (nivel 10) es también coetáneo, más o menos, de Castillo 18 y El Esquilleu. 
Nuevas dataciones para el Paleolítico superior de Cueva Morin...

Gráfico 1. Dataciones para la Transición de Catabria. M: Musteriense; AA: Auriñaciense de Transición; Au: Auriñaciense arcaico. Fuentes: La

Viña (Fortea, 1999); Castillo (Cabrera et alli, 1996); Labeko Koba (Arrizabalaga \& Altuna, 2000); El Esquilleu (Carrión, 2002)

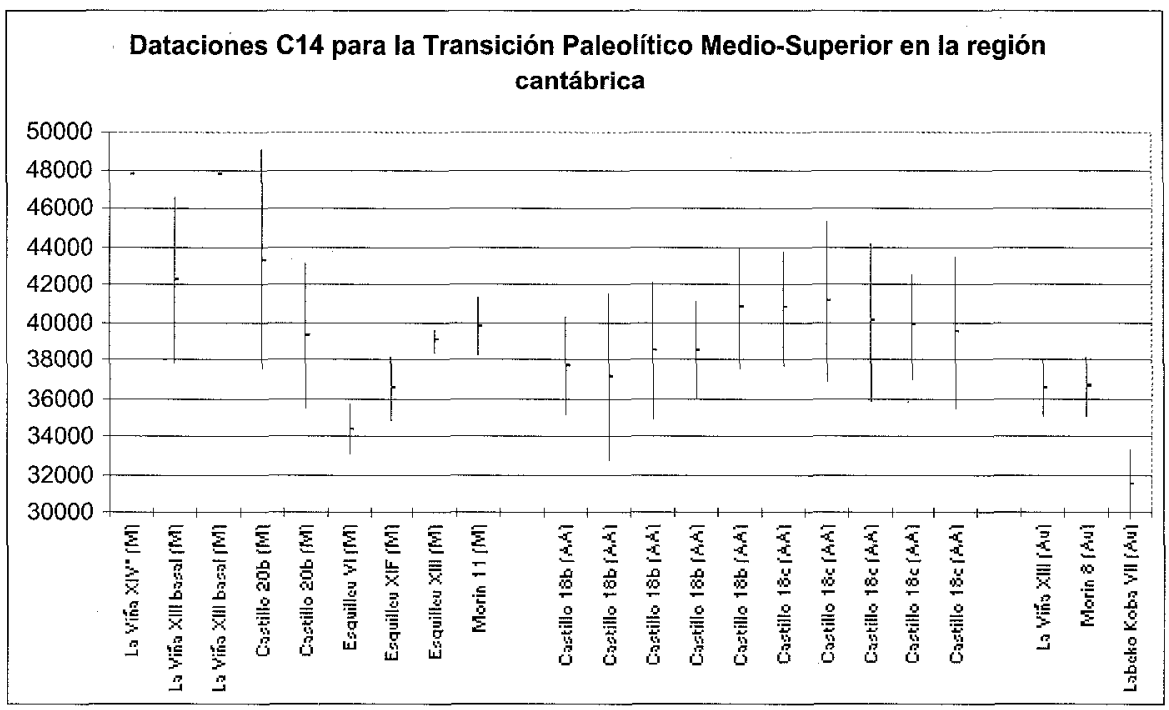

No es objetivo de este trabajo dar soluciones a estas interrogantes, pero considerábamos que sí lo era plantearlas, así como constatar el complejo e interesante escenario que se vislumbra en la Transición entre el Paleolítico medio y superior en la región cantábrica.

Agradecimientos

Agradecemos al Proyecto OICYT BHA2000-0200 y a la Consejería de Cultura, Turismo y Deporte del Gobierno de Cantabria el habernos permitido la extracción de muestras y s'ı análisis dentro del marco del proyecto interdisciplinar arriba expuesto.

\section{BIBLIOGRAFÍA}

Arrizabalaga, A. (1995): La industria lítica del Paleolítico Superior Inicial en el Oriente Cantábrico. Tesis Doctoral. Univ. País Vasco, p.1000.

Arrizabalaga, A. \& Altuna, J. (2000): Labeko Koba (País Vasco). Hienas y humanos en el Paleolítico Superior. Munibe, $52: 193-343$.

BeRnALDO DE QUIRós, F. (1982): Los Inicios del Paleolítico Superior. Centro de Investigación y Museo de Altamira, 8, $347 \mathrm{p}$. 
Cabrera, V.; Valladas, H.; Bernaldo de Quirós, F. \& Hoyos, M. (1996): La transition Paléolithique moyen-Paléolithique supérieur a El Castillo (Cantabrie): nouvelles datations por le carbone-14. Comptes Rendues de I'Academie des Sciences de Paris.

Cabrera Valdés, V.; Maíllo Fernández, J. M. \& Bernaldo De Quirós, F. (2000): Esquemas operativos laminares en el Musteriense final de la cueva del Castillo (Puente Viesgo, Cantabria). Espacio, Tiempo y Forma, Serie I, 13: 51-78.

Carballo, J. (1923) : Excavaciones en la cueva del Rey, en Villanueva (Santander). Junta Superior de Excavaciones y Antigüedades, 9, 40 p, IX lám.

González Echegaray, J. \& Freeman, L.G. (1971) : Cueva Morin. Publicaciones del Patronato de las cuevas prehistóricas de la provincia de Santander, VI, $452 \mathrm{p}$.

González Echegaray, J. \& Freeman, L.G. (1973) Cueva Morin. Publicaciones del Patronato de las cuevas prehistóricas de la provincia de Santander, X, $304 \mathrm{p}$.

González Echegaray, J. \& Freeman, L.G. (1978) : Vida y muerte en cueva Morin. Institución Cultural de Cantabria. $357 \mathrm{p}$.

Má́llo Fernández, J. M. (e. p.): El Fenómeno laminar del Paleolítico Medio: el ejemplo de Cueva Morin. Espacio, Tiempo y Forma, 14.

Maíllo Fernández, J. M. (2003): La Transición Paleolítico Medio-Superior en Cantabria: análisis tecnológico de la industria lítica de Cueva Morin. Tesis Doctoral, UNED, $547 \mathrm{p}$.

StuckENRATH, R. (1978): Dataciones de Carbono 14. En González Echegaray \& Freeman: Vida $y$ muerte en Cueva Morin: 215.

VeGa del Sella, CONDE DE LA (1921): El Paleolítico de Cueva Morin (Santander) y Notas para la climatología Cuaternaria. Comisión de Investigaciones Paleontológicas y Prehistóricas, Memoria 29, $168 \mathrm{p}$. 